\title{
Landau's and Rado's Theorems and Partial Tournaments
}

\author{
Richard A. Brualdi and Kathleen Kiernan \\ Department of Mathematics \\ University of Wisconsin \\ Madison, WI 53706 \\ \{brualdi, kiernan\}@math.wisc.edu
}

Submitted: Sep 30, 2008; Accepted: Jan 18, 2009; Published: Jan 23, 2009

Mathematics Subject Classifications: 05C07,05C20,05C50.

\begin{abstract}
Using Rado's theorem for the existence of an independent transversal of family of subsets of a set on which a matroid is defined, we give a proof of Landau's theorem for the existence of a tournament with a prescribed degree sequence. A similar approach is used to determine when a partial tournament can be extended to a tournament with a prescribed degree sequence.
\end{abstract}

Mathematics Subject Classifications: 05C07,05C20,05C50.

\section{Introduction}

A tournament of order $n$ is a digraph obtained from the complete graph $K_{n}$ of order $n$ by giving a direction to each of its edges. Thus, a tournament $T$ of order $n$ has $\left(\begin{array}{l}n \\ 2\end{array}\right)$ (directed) edges. The sequence $\left(r_{1}, r_{2}, \cdots, r_{n}\right)$ of outdegrees of the vertices $\{1,2, \ldots, n\}$ of $T$, ordered so that $r_{1} \leq r_{2} \leq \cdots \leq r_{n}$, is called the score sequence of $T$. The sequence of indegrees of the vertices of $T$ is given by $\left(s_{1}=n-1-r_{1}, s_{2}=n-1-r_{2}, \ldots, s_{n}=n-1-r_{n}\right)$ and satisfies $s_{1} \geq s_{2} \geq \cdots \geq s_{n}$. In the tournament $T^{\prime}$ obtained from $T$ by reversing the direction of each edge, the indegree sequence and outdegree sequence are interchanged; the score vector of $T^{\prime}$ equals $\left(s_{1}, s_{2}, \ldots, s_{n}\right)$ with the $s_{i}$ in nonincreasing order.

\section{Landau's theorem from Rado's theorem}

Landau's theorem characterizes score vectors of tournaments. 
Theorem 2.1 (Landau's theorem) The sequence $r_{1} \leq r_{2} \leq \cdots \leq r_{n}$ of integers is the score sequence of a tournament of order $n$ if and only if

$$
\sum_{i=1}^{k} r_{i} \geq\left(\begin{array}{l}
k \\
2
\end{array}\right) \quad(k=1,2, \ldots, n)
$$

with equality for $k=n$.

Note that (1) is equivalent to

$$
\sum_{i \in K} r_{i} \geq\left(\begin{array}{c}
|K| \\
2
\end{array}\right) \quad(K \subseteq\{1,2, \ldots, n\}) .
$$

There are several known short proofs of Landau's theorem (see $[2,3,4,7,8]$ ). In this section we give a short proof of Landau's theorem using Rado's theorem (see $[5,6]$ ) for the existence of an independent transversal of a finite family of subsets of a set $X$ on which a matroid is defined.

Let $\mathbf{M}$ be a matroid on $X$ with rank function denoted by $\rho(\cdot)$. (We assume that the reader is familiar with the very basics of matroid theory, which can be found e.g. in [6].) Let $\mathcal{A}=\left(A_{1}, A_{2}, \ldots, A_{n}\right)$ be a family of $n$ subsets of $X$. A transversal of $\mathcal{A}$ is a set $S$ of $n$ elements of $X$ which can be ordered as $x_{1}, x_{2}, \ldots, x_{n}$ so that $x_{i} \in A_{i}$ for $i=1,2, \ldots, n$. The transversal $S$ is an independent transversal of $\mathcal{A}$ provided that $S$ is an independent set of the matroid $\mathbf{M}$.

Theorem 2.2 (Rado's theorem) The family $\mathcal{A}=\left(A_{1}, A_{2}, \ldots, A_{n}\right)$ of subsets of the set $X$ on which a matroid $\mathbf{M}$ is defined has an independent transversal if and only if

$$
\rho\left(\cup_{i \in K} A_{i}\right) \geq|K| \quad(K \subseteq\{1,2, \ldots, n\}) .
$$

Proof of Landau's theorem using Rado's theorem. The necessity of (1) is obvious. Now assume that (1) holds. Let $X=\{(i, j) ; 1 \leq i, j \leq n, i \neq j\}$. Consider the matroid $\mathbf{M}$ on $X$ whose circuits are the $\left(\begin{array}{l}n \\ 2\end{array}\right)$ disjoint sets $\{(i, j),(j, i)\}$ of two pairs in $X$ with $i \neq j$. Thus, a subset $E$ of $X$ is independent if and only if it does not contain a symmetric pair $(i, j),(j, i)$ with $i \neq j$. We have $\rho(X)=\left(\begin{array}{l}n \\ 2\end{array}\right)$. Let $\mathcal{A}=\left(A_{1}, A_{2}, \ldots, A_{n}\right)$ be the family of subsets of $X$ where

$$
A_{i}=\{(i, j): 1 \leq j \leq n, j \neq i\} \quad(i=1,2, \ldots, n) .
$$

Let $r_{1}, r_{2}, \ldots, r_{n}$ be a sequence of nonnegative integers with $r_{1}+r_{2}+\cdots+r_{n}=\left(\begin{array}{l}n \\ 2\end{array}\right)$. There exists a tournament with score sequence $r_{1}, r_{2}, \ldots, r_{n}$ if and only if there exists $P_{1}, P_{2}, \ldots, P_{n}$, with $P_{i} \subseteq A_{i}$ and $\left|P_{i}\right|=r_{i}(1 \leq i \leq n)$, such that $P=P_{1} \cup P_{2} \cup \cdots \cup P_{n}$ is an independent set of $\mathbf{M}$, equivalently, if and only if the family

$$
\mathcal{A}^{\prime}=(\underbrace{A_{1}, \ldots, A_{1}}_{r_{1}}, \underbrace{A_{2}, \ldots, A_{2}}_{r_{2}}, \ldots, \underbrace{A_{n}, \ldots, A_{n}}_{r_{n}})
$$


has an independent transversal: The desired tournament has vertices $1,2, \ldots, n$ and an edge from $i$ to $j$ if and only $(i, j)$ is in $P_{i}$. The independence of $P$ then implies that there is no edge from $j$ to $i$.

It follows from Rado's theorem that $\mathcal{A}^{\prime}$ has an independent transversal provided that

$$
\rho\left(\cup_{i \in K} A_{i}\right) \geq \sum_{i \in K} r_{i} \quad(K \subseteq\{1,2, \ldots, n\}) .
$$

From the definition of $\mathbf{M}$ we see that

$$
\rho\left(\cup_{i \in K} A_{i}\right)=\left(\begin{array}{l}
k \\
2
\end{array}\right)+k(n-k),
$$

where $k=|K|$. By (5), the rank of $\cup_{i \in K} A_{i}$ depends only on $k=|K|$. By the monotonicity assumption on the $r_{i}, \sum_{i \in K} r_{i}$ is largest when $K=\{n-k+1, \ldots, n\}$. Thus, (4) is equivalent to

$$
\left(\begin{array}{l}
k \\
2
\end{array}\right)+k(n-k) \geq \sum_{i=n-k+1}^{n} r_{i} .
$$

Since $\sum_{i=1}^{n} r_{i}=\left(\begin{array}{l}n \\ 2\end{array}\right)$, (6) becomes

$$
\sum_{i=1}^{n-k} r_{i} \geq\left(\begin{array}{l}
n \\
2
\end{array}\right)-\left(\begin{array}{l}
k \\
2
\end{array}\right)-k(n-k) .
$$

It follows that (4) is equivalent to

$$
\sum_{i=1}^{p} r_{i} \geq\left(\begin{array}{l}
n \\
2
\end{array}\right)-\left(\begin{array}{c}
n-p \\
2
\end{array}\right)-p(n-p) \quad(p=1,2, \ldots, n) .
$$

A simple calculation shows that

$$
\left(\begin{array}{l}
n \\
2
\end{array}\right)-\left(\begin{array}{c}
n-p \\
2
\end{array}\right)-p(n-p)=\left(\begin{array}{l}
p \\
2
\end{array}\right)
$$

and Landau's theorem follows from (8).

\section{Completions of partial tournaments}

Let $G \subseteq K_{n}$ be a graph on $n$ vertices. A digraph obtained from $G$ by giving a direction to each of its edges is called an oriented graph or a partial tournament of order $n$. Given a partial tournament $T^{\prime}$ and a sequence of nonnegative integers $r_{1}, r_{2}, \ldots, r_{n}$, it is possible to use Rado's theorem to establish necessary and sufficient conditions for $T^{\prime}$ to be extendable to a tournament $T$ with score sequence $r_{1}, r_{2}, \ldots, r_{n}$. Thus we seek to complete the partial tournament $T^{\prime}$ to a tournament $T$ with a prescribed score sequence. Rado's theorem can also be used to characterize when such a completion is possible. 
Let $T^{\prime}$ be a partial tournament of order $n$ with outdegree sequence $s_{1}, s_{2}, \ldots, s_{n}$. Let $r_{1}, r_{2}, \ldots, r_{n}$ be a sequence of nonnegative integers with $\sum_{i=1}^{n} r_{i}=\left(\begin{array}{l}n \\ 2\end{array}\right)$. (Now we make no monotone assumption on the $r_{i}$ or the $s_{i}$.) An obvious necessary condtion for $T^{\prime}$ to be completed to a tournament with score sequence $r_{1}, r_{2}, \ldots, r_{n}$ is that $s_{i} \leq r_{i}$ for $i=1,2, \ldots, n$, and we assume these inequalities hold. There are two ways to determine when a completion of $T^{\prime}$ to a tournament with score sequence $r_{1}, r_{2}, \ldots, r_{n}$ is possible.

The first way is to take $X=\{(i, j): 1 \leq i, j \leq n, i \neq j\}$ as before, and to consider the matroid $\mathbf{M}^{\prime}$ whose circuits are the singleton pairs $\{(i, j)\}$ and $\{(j, i)\}$ if there is an edge from $i$ to $j$ in $T^{\prime}$ (thus an edge in $T$ determines two loops of $\mathbf{M}^{\prime}$ ), and the pairs $\{(i, j),(j, i)\}$ for all distinct $i$ and $j$ such that there is no edge in $T^{\prime}$ between $i$ and $j$ (in either of the two possible directions). We note that in this matroid $\mathbf{M}^{\prime}$,

$$
\rho^{\prime}(X)=\left(\begin{array}{l}
n \\
2
\end{array}\right)-\sum_{i=1}^{n} s_{i}
$$

Define the family $\mathcal{A}=\left(A_{1}, A_{2}, \ldots, A_{n}\right)$ as in (3) and the family

$$
\mathcal{A}^{\prime \prime}=(\underbrace{A_{1}, \ldots, A_{1}}_{r_{1}-s_{1}}, \underbrace{A_{2}, \ldots, A_{2}}_{r_{2}-s_{2}}, \ldots, \underbrace{A_{n}, \ldots, A_{n}}_{r_{n}-s_{n}}) \text {. }
$$

We have

$$
\sum_{i=1}^{n}\left(r_{i}-s_{i}\right)=\left(\begin{array}{l}
n \\
2
\end{array}\right)-\sum_{i=1}^{n} s_{i}
$$

The partial tournament $T^{\prime}$ can be completed to a tournament with score sequence $r_{1}, r_{2}, \ldots, r_{n}$ if and only if the family $\mathcal{A}^{\prime \prime}$ has an independent transversal. It follows from Rado's theorem that $\mathcal{A}^{\prime \prime}$ has an independent transversal if and only if

$$
\rho^{\prime}\left(\cup_{i \in K} A_{i}\right) \geq \sum_{i \in K}\left(r_{i}-s_{i}\right) \quad(K \subseteq\{1,2, \ldots, n\}) .
$$

For $K \subseteq\{1,2, \ldots, n\}$, let $\gamma(K)$ equal the number of edges of $T^{\prime}$ at least one of whose vertices belongs to $K$. We easily calculate that

$$
\rho^{\prime}\left(\cup_{i \in K} A_{i}\right)=\left(\begin{array}{c}
|K| \\
2
\end{array}\right)+|K|(n-|K|)-\gamma(K) .
$$

We thus obtain the following generalization of Landau's theorem. ${ }^{1}$

Theorem 3.1 Let $T^{\prime}$ be a partial tournament with outdegree sequence $s_{1}, s_{2}, \ldots, s_{n}$. Let $r_{1}, r_{2}, \ldots, r_{n}$ be a sequence of nonnegative integers with $s_{i} \leq r_{i}$ for $i=1,2, \ldots, n$. Then $T^{\prime}$ can be completed to a tournament with score sequence $r_{1}, r_{2}, \ldots, r_{n}$ if and only if

$$
\left(\begin{array}{c}
|K| \\
2
\end{array}\right)+|K|(n-|K|)-\gamma(K) \geq \sum_{i \in K}\left(r_{i}-s_{i}\right) \quad(K \subseteq\{1,2, \ldots, n\} .
$$

\footnotetext{
${ }^{1}$ Landau's theorem is the special case where $T^{\prime}$ has no edges.
} 
As a referee observed, because of the presence of the quantity $\gamma(K)$, whether or not the inequalities (10) in Theorem 3.1 are satisfied depends on the initial labeling of the vertices of $T^{\prime}$. These conditions may not be satisfied according to one labeling but satisfied according to another.

A second, but basically equivalent, way to approach the proof of Theorem 3.1 is to start with the set

$$
Y=X \backslash\left\{(i, j):(i, j) \text { or }(j, i) \text { is an edge of } T^{\prime}\right\},
$$

and the matroid $\left.\mathbf{M}\right|_{Y}$ on $Y$ obtained by restricting $\mathbf{M}$ to $Y$. If we define the family $\mathcal{B}=\left(B_{1}, B_{2}, \ldots, B_{n}\right)$ of subsets of $Y$ by $B_{i}=A_{i} \cap Y$ for $i=1,2, \ldots, n$, and then apply Rado's theorem to

$$
\mathcal{B}^{\prime}=(\underbrace{B_{1}, \ldots, B_{1}}_{r_{1}-s_{1}}, \underbrace{B_{2}, \ldots, B_{2}}_{r_{2}-s_{2}}, \ldots, \underbrace{B_{n}, \ldots, B_{n}}_{r_{n}-s_{n}})
$$

we again obtain a proof of Theorem 3.1.

As a corollary of Theorem 3.1 we obtain the main results in [1]. If $n$ is an odd integer, a regular tournament of order $n$ is a tournament with score sequence

$$
\underbrace{\frac{n-1}{2}, \frac{n-1}{2}, \ldots, \frac{n-1}{2}}_{n} \text {. }
$$

If $n$ is an even integer, a nearly regular tournament of order $n$ is a tournament with score sequence

$$
\underbrace{\frac{n}{2}, \ldots, \frac{n}{2}}_{\frac{n}{2}}, \underbrace{\frac{n}{2}-1, \ldots, \frac{n}{2}-1}_{\frac{n}{2}}
$$

Corollary 3.2 Let $T^{\prime}$ be a partial tournament with outdegree sequence $s_{1}, s_{2}, \ldots, s_{n}$ where $s_{1} \geq s_{2} \geq \cdots \geq s_{n}$. If $n$ is odd, then $T^{\prime}$ can be completed to a regular tournament provided that

$$
s_{i} \leq \frac{n+1}{2}-i, \quad\left(i=1,2, \ldots, \frac{n+1}{2}\right) .
$$

If $n$ is even, then $T^{\prime}$ can be completed to a nearly regular tournament of order $n$ provided that

$$
s_{i} \leq \frac{n}{2}-i+1, \quad\left(i=1,2, \ldots, \frac{n}{2}\right)
$$

Proof. First suppose that $n$ is odd and that (11) holds. Then $s_{i}=0$ for $i=(n+$ $1) / 2,(n+3) / 2, \ldots, n$. Hence, there are no edges in $T^{\prime}$ from a vertex in $\{(n+1) / 2,(n+$ $3) / 2, \ldots, n\}$ to $\{1,2, \ldots,(n-1) / 2\}$. It follows from Theorem 3.1 that $T^{\prime}$ can be completed to a regular tournament provided that

$$
\left(\begin{array}{c}
|K| \\
2
\end{array}\right)+|K|(n-|K|)-\gamma(K) \geq|K|\left(\frac{n-1}{2}\right)-\sum_{i \in K} s_{i} \quad(K \subseteq\{1,2, \ldots, n\},
$$


that is, provided that

$$
\left(\begin{array}{c}
|K| \\
2
\end{array}\right)+|K|(n-|K|)-\left(\gamma(K)-\sum_{i \in K} s_{i}\right) \geq|K|\left(\frac{n-1}{2}\right) \quad(K \subseteq\{1,2, \ldots, n\}) .
$$

The quantity $\gamma^{*}(K):=\gamma(K)-\sum_{i \in K} s_{i}$ equals the number of edges of $T^{\prime}$ with initial vertex in the complement $\bar{K}$ of $K$ and terminal vertex in $K$. Simplifying (13), we get

$$
\frac{|K||\bar{K}|}{2} \geq \gamma^{*}(K)
$$

Since the lefthand side of (14) is symmetric in $K$ and $\bar{K}$, we need only verify it for $|K| \leq(n+1) / 2$. It follows from (11) that for $|K| \leq(n+1) / 2$,

$$
\gamma^{*}(K) \leq \sum_{i=1}^{|K|}\left(\frac{n+1}{2}-i\right)=\frac{|K|(n-|K|)}{2}
$$

Hence, $T^{\prime}$ can be completed to a regular tournament.

A similar proof works when $n$ is even.

\section{References}

[1] L. Beasley, D. Brown, and K. B. Reid, Extending partial tournaments, Mathematical and Computer Modelling, to appear.

[2] R. A. Brualdi, Combinatorial Matrix Classes, Cambridge U. Press, Cambridge, 2006, $34-35$.

[3] J.R. Griggs and K.B. Reid, Landau's theorem revisited, Australasian J. Combinatorics, 20 (1999), 19-24.

[4] E. S. Mahmoodian, A critical case method of proof in combinatorial mathematics, Bull. Iranian Math Soc., No. 8 (1978),1L-26L.

[5] L. Mirsky, Transversal Theory, Oxford University Press, Oxford, 1971, 93-95.

[6] J. Oxley, Matroid Theory, The Clarendon Press, Oxford University Press, New York, 1992.

[7] K.B. Reid, Tournaments: scores, kings, generalizations and special topics, Congressus Numerantium, 115 (1996), 171-211.

[8] C. Thomassen, Landau's characterization of tournament score sequences, The Theory and Application of Graphs (Kalamazoo, Michigan 1980), Wiley, New York, 1963, 589-591. 